\title{
AN ENERGY-EFFICIENT RANDOM FINGERPRINT AND VERIFICATION FOR WIRELESS SENSOR NETWORKS AUTHENTICATION PROTOCOLS
}

\author{
Youssou Faye ${ }^{1}$, Hervé Guyennet ${ }^{2}$ and Pierre Cyrille Héam ${ }^{2}$ \\ ${ }^{1}$ Department of Computer Sciences, University Assane Seck of Ziguinchor, Senegal \\ ${ }^{2}$ Department of Computer Sciences, University of Franche Comte, France
}

\begin{abstract}
Sensor devices are limited resources (memory storage, energy, power computation and transmission), thus providing security mechanisms with lightweight resources is a very challenge in Wireless Sensors Network (WSNs). Recently, we show that, Vaidya and al.'s Improved Robust scheme for Dynamic User Authentication in WSNs is vulnerable to the risk of forgery attacks and Denial-of-Service (DoS) and we have proposed a lightweight solution which is adequate for resource constrained sensor networks. In this paper, we derive from password a much shorter string that could serve as random fingerprint to improve Login and Authentication phase. After having taken note that the security analysis of authentication protocols has already demonstrated through hash function properties and computational complexity, we propose an evaluation by experimenting firstly on the real sensor platform SensLab to measure energy consumption. After, we verify secrecy and authentication properties by the widely accepted AVISPA (Automated Validation of Internet Security Protocols and Applications) tool and validate protocols through simulation based on attacks.
\end{abstract}

\section{KEYWORDS}

Wireless Sensor Networks, Authentication, Password, Verification of Authentication Protocols

\section{INTRODUCTION}

Wireless Sensors Network are often deployed in hostile environments which make them more vulnerable to various attacks. In this context access control become necessary (Faye, 2011). In WSNs critical applications, network should be protected by preventing unauthorized users from gaining the information. User Authentication is the most basic used solution in traditional networks. Nowadays in WSNs, it has been adequately addressed with sensors limited resources. Many researchers have proposed a number of WSN authentication schemes (Vaidya, 2009), (Faye, 2012), (Wong, 2006), (Das, 2016), (Zhang, 2018), (Li, 2018). Recently, Vaidya and al. proposed an Improved Robust Dynamic User Authentication Scheme for WSNs (Vaidya, 2009). Only four phases are used in this scheme, namely, Registration Phase (RP), Login Phase (LP), Authentication Phase (AP), and Password-changing Phase (PP). In (Faye, 2012), authors shown that Vaidya and al.'s scheme is insecure and cannot prevent from DoS attacks. Hash function properties and computational complexity are generally used in password-based authentication schemes for performance evaluation and security (Kumari, 2015).

In this paper, to overcome the challenge of optimizing energy consumption in (Faye, 2012) and all schemes using the same approach during the Login Phase and the Authentication Phase which are the most time-consuming part of transmission, we reduce the bits transmitted by deriving from password a much shorter string that could serve as randomized fingerprinting function to improve LP and AP phases.

In the rest of the paper, Section 2 presents related works. The lack of energy reduction in (Faye, 2012). The proposed randomized fingerprinting technique is described in Section 4 and its security is discussed in Section 5. The implementation on SensLAB is done in section 6, and after, a security check and validation with AVISPA are made in section 7. Finally, we conclude the paper in section 8. 


\section{RELATED WORKS}

For monitoring access control in many existing applications and computer systems, User Authentication (UA) has been the most widely used basic solution. Initially, traditional UA schemes (Awasthi, 2003), (Hwuang,2002), (Lee, 2002), (Shen, 2003),(Sun, 2000) based on (Lamport, 1981) scheme are quite interesting to examine various works on smart cards, they are based on static login ID and use the techniques of Password-based authentication for remote UA, on which a verification table is no longer required in the remote system. For existing Password-based authentication solutions, some schemes use the technique of a weak password, which facilitates the memorization of the password. However, they are very difficult to apply in WSN because it is based on public-key cryptographic techniques. The others (Awasthi, 2003), (Das, 2004) use strong-password and lead lighter computational because of using only one-way hash function and exclusive-OR operation (XOR). That's made it feasible to be adapted into the WSN environment. Their disadvantage lies in the difficulty of memorizing the password. (Lee, 2005) have shown that solutions like (Das, 2004) based on a dynamic login are vulnerable to reply attacks and modified login message attacks. They also proposed an improved UA scheme, which offers a low computation cost based on one-way hash functions. For better adaptation in WSN environment, (Wong, 2006) improves Lee's framework to provide the dynamic UA. (Vaidya, 2009), propose a variation of strong-password based solution proposed by (Wong, 2006). This scheme claimed to provide protection against the replay attacks of login message and forgery attacks. In (Faye, 2012), authors have shown that (Vaidya, 2009) is vulnerable to DoS attack and forgery attacks, and have proposed a solution. Using the same approach, (Xue, 2013) proposed mutual authentication based on the same technique of hash functions and XOR operations. (Jiang, 2015) points out vulnerabilities in Xue et al.'s scheme and proposed a two-factor authentication solution. (Das, 2016) showed that Jiang et al. does not produce proper authentication during the login and authentication phases and proposes a solution based on three authentication factors. Recently, some works in WSN and IoT field, such as (Zhang, 2018), (Ling, 2017), (Li, 2018) are also use technique of one-way hash function and exclusive-OR operation and the handshake structure of Registration Phase (RP), Login Phase (LP) and Authentication Phase (AP) on multi-hop communication. For an evaluation of the energy cost, all solutions use almost the same comparative approach based on analytical computational and communication complexity (Kumari, 2015).

\section{LACK OF ENERGY REDUCTION}

In order to better express the lack of energy reduction on schemes (Vaidya, 2009),(Wong, 2006), (Faye, 2012), (Xue, 2013), (Zhang, 2018), (Li, 2018), we will review the protocol in (Faye, 2012) as an example..

\subsection{Review of Protocol in (Faye, 2012)}

We briefly describe schemes in Vaidya et al, and Faye et al. The notations involved are listed in Table 1.

Table 1. Notations

\begin{tabular}{ll}
\hline Symbols & Description \\
\hline UD & Users Device such PDA, PC \\
GW and LN & Respectively Registration Sensor Gateway and Sensor login Node \\
$\mathrm{H}()$ and $\mathrm{H}_{\mathrm{p}}()$ & Respectively One-way hash function and $\mathrm{H}()$ mod $(\mathrm{p})$ \\
$\oplus$ and $\|$ & Respectively Exclusive-or (XOR) operation and Concatenation \\
Succ_Reg & Successful Registration message \\
Acc_login & Accept login message \\
Succ_Chenge & Successful Changes message \\
$\mathrm{X}$ & Secret key known to the GW \\
$\mathrm{UID}$ and $\mathrm{PW}$ & Respectively Users identity and Password chosen by user \\
$\mathrm{TS}$ & Registration time \\
$\mathrm{t}, \mathrm{T}_{0}, \mathrm{~T}_{1}, \mathrm{~T}_{2}, \mathrm{~T}_{3}$ & Current time recorded by one of the nodes \\
$\Delta \mathrm{T}$ & Allowed time interval for transmission delay \\
$\rightarrow$ & transmission of message \\
$\mathrm{P}$ & a prime number \\
\hline
\end{tabular}




\subsubsection{Registration Phase}

It is important to note that, there is one-hop communication between user's mobile device and the LN during the login phase, and a multiple hops communication between the $\mathrm{LN}$ and the gateway node.

RP1 - In (Vaidya, 2009), it is not necessary to compute vpw $=\mathrm{H}(\mathrm{PW})$, because it is as vulnerable as the PW. Thus, in (Faye, Niang, \& Guyennet, 2012), user UD can only choose a PW.

RP2 - At a time TS, the UD submits its identity UID and the password PW to the GW in secure mode.

RP3 - The GW computes $\mathrm{X}=\mathrm{H}(\mathrm{UID} \| \mathrm{x}$ ) and replies to UD for successful registration, stores (UID, H(PW), $\mathrm{X}, \mathrm{TS}$ ), and distributes (UID, $\mathrm{X}, \mathrm{H}(\mathrm{PW}), \mathrm{TS})$ to sensor nodes, which can provide a login interface to users.

\subsubsection{The Login Phase}

LP1 - The user computes $\mathrm{A}=\mathrm{H}(\mathrm{H}(\mathrm{PW}) \| \mathrm{t}$ ) and submits (UID, $\mathrm{A}, \mathrm{t})$ to a login node

LP2 - After receive the login request at time T0, the LN checks its lookup table to verify if UID is a valid. The login request is rejected if it is not.

if

LP3 - The login node computes $A^{\prime}=H(H(P W) \| t)$, checks if $A=A^{\prime}$ and $T_{0}-t \geq \triangle T$ (the password is valide

$\left.A=A^{\prime}\right)$. The login request is rejected if it is not. Otherwise, the login node retrieves the corresponding

A, computes $\mathrm{C}_{\mathrm{K}}=\left(\mathrm{X} \oplus \mathrm{A} \oplus \mathrm{T}_{0}\right)$ and computes $\mathrm{t}^{\prime}=\mathrm{H}^{2}(\mathrm{PW}) \bigoplus$. The expression $\mathrm{H}^{\mathrm{n}}(\mathrm{PW})$ denotes the application of $n$-times hash operations on PW. That is, $\mathrm{H}^{\mathrm{n}}(\mathrm{PW})=\mathrm{H}\left(\mathrm{H}^{\mathrm{n}-1}(\mathrm{PW})\right)$.

\subsubsection{Authentication Phase}

AP1 -The gateway checks the validity of UID and t. The login request is rejected if they are not valid.

Otherwise, the GW Computes $\mathrm{t}=\mathrm{t}^{\prime} \oplus \mathrm{H}^{2}(\mathrm{PW}), \quad$ checks if $\mathrm{T}_{1}-\mathrm{T}_{0} \geq \triangle \mathrm{T} ; \mathrm{T}_{0}-\mathrm{t} \geq \triangle \mathrm{T}$. If the condition is satisfied, then the login request is a replay message and is rejected. The gateway retrieves the corresponding $\mathrm{H}(\mathrm{PW})$ and $\mathrm{A}$, computes $\mathrm{A}^{\prime}=\mathrm{H}(\mathrm{H}(\mathrm{PW}) \| \mathrm{t})$ and $\mathrm{C}_{\mathrm{K}^{\prime}}=\left(\mathrm{X} \oplus \mathrm{A}^{\prime} \oplus \mathrm{T}_{0}\right)$. The login request is rejected if $C_{K} \neq C_{K}{ }^{\prime}$. Otherwise, the $G W$ computes $V_{M}=H\left(X\left\|A^{\prime}\right\| T_{1}\right)$. t.

AP2 -GW validates the login request, sends accept message (Access_login, $\mathrm{V}_{\mathrm{M}}, \mathrm{T}_{1}$ ) to the LN; and stores

AP3 -The LN computes $V_{M^{\prime}}$, and after checking of $V_{M}=V_{M^{\prime}}$, it calculates $Y_{K}=H\left(V_{M^{\prime}} \| T_{2}\right)$

AP4 - The LN sends (Access_login, $\mathrm{Y}_{\mathrm{K}}, \mathrm{T}_{1}, \mathrm{~T}_{2}$ ) to the user UD

AP5 - Upon receiving the message at time $T_{3}$, the UD checks if $T_{1}-T_{0} \geq \Delta T ; T_{0}-t \geq \triangle T$. If the conditions are true, then the Access login message is rejected, else, the $\mathrm{LN}$ retrieves $\mathrm{A}$, performs $\mathrm{V}_{\mathrm{M}}$ " =

$\mathrm{H}\left(\mathrm{X}\|\mathrm{A}\| \mathrm{T}_{1}\right)$ and $\quad \mathrm{Y}_{\mathrm{K}}{ }^{\prime}=\mathrm{H}\left(\mathrm{V}_{\mathrm{M}}{ }^{\prime} \| \mathrm{T}_{2}\right)$, and checks if $\mathrm{Y}_{\mathrm{K}}=\mathrm{Y}_{\mathrm{K}^{\prime}}$. If the condition holds, then the UD starts getting data, else, the accept Access login message is rejected.

\subsection{Costly communication: similar approaches to Vaidya et al. and Faye et al.}

Similar solutions Wong et al., Xue et al., Zhang et al., Li et al., to Vaidya et al. and Faye et al. use the technique of hash functions and XOR operations and are based on the handshake structure of Registration Phase (RP), Login Phase (LP) and Authentication Phase (AP) on multi-hop communication. During the login phase PL3, the $\mathrm{LN}$ after verification, calculates $\mathrm{CK}=\left(\mathrm{X} \oplus \mathrm{A} \oplus \mathrm{T}_{0}\right)$ and $\mathrm{t}^{\prime}=\mathrm{H}^{2}(\mathrm{PW}) \oplus \mathrm{t}$ and sends them to the $\mathrm{GW}$ through a multi-hop communication provided by the sensors. Since the transmission is the most energy consuming operation in wireless communication, the long string of bit $C_{K}$ is equal to the maximum number of bits between $\mathrm{X}$ and $\mathrm{A}$. Therefore, a long string of bit login or password can lead to a high energy consumption in the network. Thus finding ways to minimize the number of bits transmitted during this multi-hop communication between LN and GW without compromising security becomes necessary for energy saving. Since the length bit of timestamps is very small compared to $\mathrm{X}$ and A, we applying some standard hashing or check technique to derive from $\mathrm{X}$ and $\mathrm{A}$ a much shorter string that could serve as a fingerprint of $\mathrm{X}$ and $\mathrm{A}$. 


\section{REDUCTION OF THE BITS TRANSMITTED BY FINGERPRINT}

In order to avoid the propagation of a large number of bits through a multi-hop communication provide by sensors, we propose a technique based on the modulo operator on prime numbers. To do this, in the same way as the hash function $\mathrm{H}()$, a random prime number $p$ is generated by the $\mathrm{LN}$ and the $\mathrm{GW}$ in login phase RP1. To produce much shorter string, we use reduction modulo $p$ as the fingerprinting function $\mathrm{H}_{\mathrm{p}}()$ such as $H_{p}(x)=H(x)\left(\bmod _{p}\right)$ and where $H(x)$ is the integer represented by the bit string $x$. If $p$ is not too large, then the fingerprint function $\mathrm{Hp}()$ can be transmitted as a short string of bits. The property $\mathrm{H}_{\mathrm{p}}(\mathrm{x})=\mathrm{H}(\mathrm{x})(\bmod p)$ is checked if $x=y$ and checked with some probability if $\mathrm{x} \neq \mathrm{y}$. When $p$ becoming increasingly smaller, $\mathrm{H} p(\mathrm{x})$ becomes a more short binary sequence. Thus registration phase (RP1, RP2, RP3) and the two first steps of login phase (LP1, LP2) are not changed. However the third phase LP3 which induces a multi-hop communication through the sensors may be proceed according to the following algorithm.

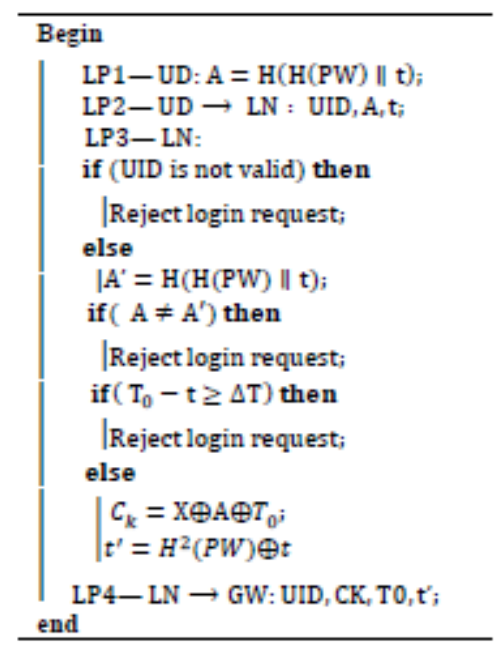

Algorithm 1. Protocol in

(Faye, Niang, \& Guyennet, 2012): LP

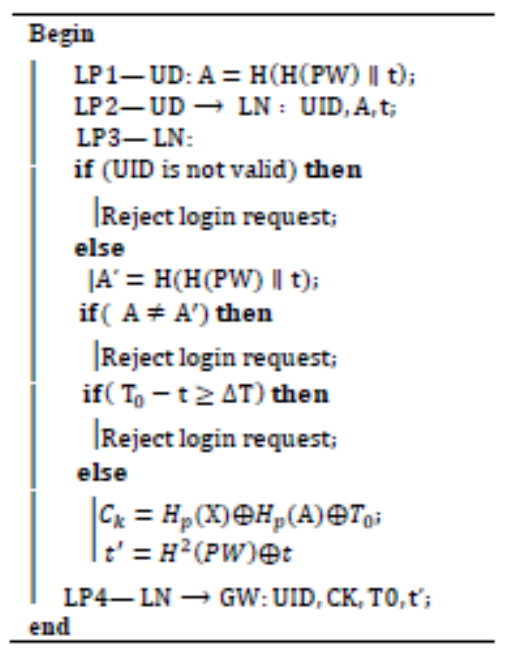

Algorithm 2. Proposed solution: LP

\section{SECURITY ANALYSIS}

This technique reduces the number of bits transmitted between the LN and the GW. The value of the prime number $\mathrm{p}$ is one of the main keys for efficiency. When $p$ is small, the number of transmitted bit length becomes more shortly but allows the possibility of a false match, in which $x$ and $y$ have the same fingerprint even though they are different. The weakness of this technique is that, if $p$ is held fixed, then there are certain pairs of strings $x$ and $y$ on which the method will always fail. A more advantageous method, which avoids such bad pairs $x$ and $y$, is to determine randomly the prime $p$ on every time the equality of two strings is to be checked, rather than to agree on $p$ in advance. This solution does not compromise security, because registration phase is not change. According to the size of prime number $\mathrm{p}$ the hash function $H_{p}()$ can have all properties of the hash function $H()$ with a very low probability (even zero) to permit the possibility of a false match in which $x$ and $y$ have the same fingerprint $\left(H_{p}(x)=H_{p}(y)\right)$ even though they are different $(\mathrm{x} \neq \mathrm{y})$. In other words $H_{p}(x) \equiv H_{p}(y)$ mod $p$. Clearly, if $x=y$ then this algorithm gives the correct answer (since $\left.H_{p}(x)=H_{p}(y)\right)$. However, if $\mathrm{x} \neq \mathrm{y}$, there is a chance that this algorithm gives a wrong answer. But the probability that the algorithm will fail is extremely small. We upper bound this probability. If $n$ is the number of bits to transmit without use reduction modulo $\mathrm{p}$ as the fingerprinting function, failure can occur only if $\mathrm{p}$ divides $|H(x)-H(y)|$. It is well known that for any prime number $\mathrm{p}>2$, if $\left|H_{p}(x)-H_{p}(y)\right| \leq 2^{n}$, there are at most $\mathrm{n}$ primes that divide $\mid \mathrm{H}(\mathrm{x})$ - $\mathrm{H}(\mathrm{y}) \mid$ (Karp, 1991). Therefore, $n$ is an upper bound on the number of primes, i.e. those that if we select the algorithm will fail. It is well known and well-studied in number theory that the number of minimum password length of 8 or more characters is equivalent to a minimum of 64 bits in 8 -bit ASCII. Figure1 shows, for 104 bits ( a password length of 12 characters) to be transmitted, the probability of failure Pr depending on the number of bits effectively transmitted based on fingerprinting function. We can notice that from a transmission of a fingerprint $\geq 24$ bits, we have a very good probability 
$\leq 0,000000292$. For a minimum password length of 8 characters ( 64 bits to be transmitted), a transmission of a fingerprint $=24$ bits have a very good probability of 0,000000318 . Table 2 shows the probability of failure $\mathrm{P}$ $r$ for a password length of 64 bits and 104 bits based on fingerprinting length for a set of values between 1 and 40 .
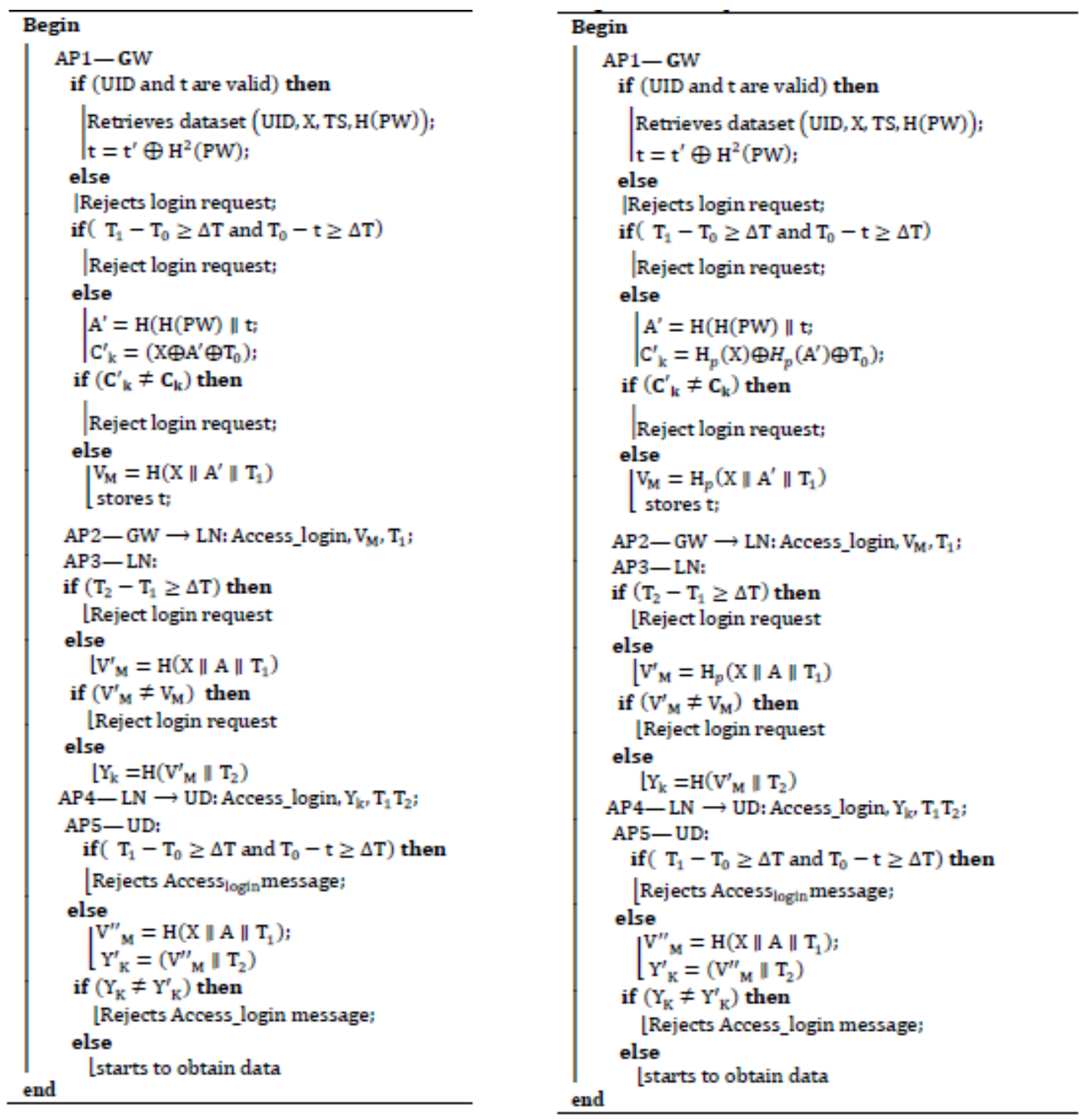

Algorithm 3. Protocol in (Faye, Niang, Guyennet, 2012): AP

Algorithm 4. Proposed solution AP

Algorithm 3: Protocol in (Faye, Niang, Guyennet, 2012): AP Algorithm 4: Algorithm 4: Proposed solution APprimes less than $z$ is asymptotic to $\frac{z}{\ln (z)}$, i.e. the number of primes up to $z$ is about (Karp, 1991). Then we can deduce that the probability of failure (false match i.e. $\mathrm{x} \neq \mathrm{y}$, while $\mathrm{H}_{\mathrm{p}}(\mathrm{x})=\mathrm{H}_{\mathrm{p}}(\mathrm{y})$ ) is equal to $\operatorname{Pr}[$ failure $]=\operatorname{Pr}[H(x) \bmod p=H(y) \bmod p]$ is $\operatorname{Pr}=\frac{n}{\frac{2^{n}}{\ln \left(2^{n}\right)}}$. This probability varies depending on the number of reduced bits according to the size of the prime $\mathrm{p}$. if the prime $\mathrm{p}$ is becoming increasingly smaller, the transmitted bits are reduced and the probability of failure $P r$ increases. Thus, there is a need to find a compromise between the number of transmitted bits and the probability Pr. It is important to estimate the length of hash values for applications. In variety of tools and security templates, the password must be at least 8 characters. If we take account human memory limitations, we shall assume a secure password can be composed by a minimum of 8 characters, where each character can be any of the 95 characters readily represented with keyboard. 


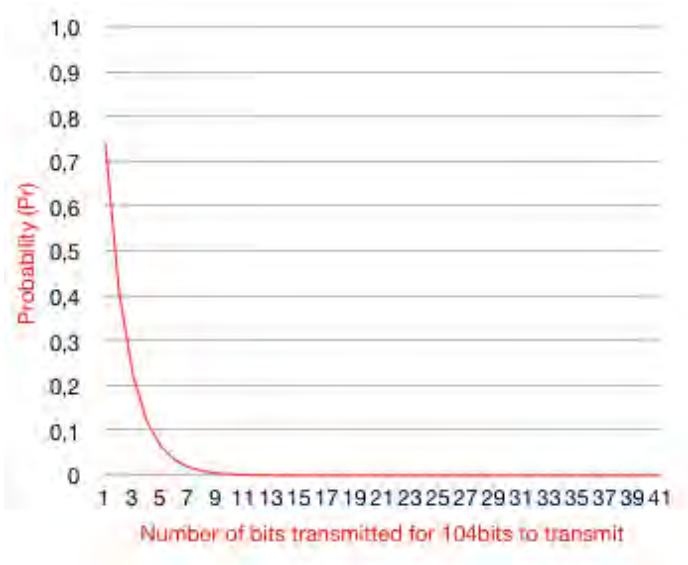

Figure 1. Probability of failure depending on the number of bits transmitted / 104bits to transmit

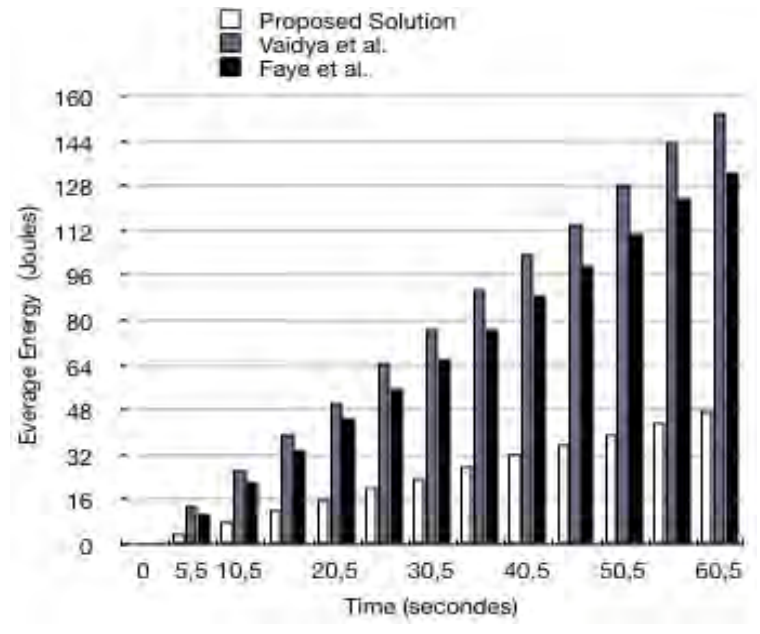

Figure 2. Energy consumption

Table 2. Pr for a set of values of fingerprint length

\begin{tabular}{llllll}
\hline Fingerprint & 1 & 4 & 10 & 19 & 32 \\
\hline Pr for 104 bits & $\mathbf{0 , 7 3 9}$ & $\mathbf{0 , 1 2 0}$ & $\mathbf{0 , 0 0 2 7 5}$ & $\mathbf{7 9 4 . 1 0}^{-8}$ & $142.10^{-11}$ \\
Pr for 64 bits & $\mathbf{0 , 7 5 4}$ & $\mathbf{0 , 1 2 6}$ & $\mathbf{0 , 0 0 2 9 3}$ & $\mathbf{8 6 0 . 1 0}^{-8}$ & $155.10^{-11}$ \\
\hline
\end{tabular}

In this case, random prime p can be generated by LN and GW from the forty least significant bits of the password, and where the twentieth bit is set to 1 . The random prime is the nearest prime value got from the resulting binary suite.

\section{IMPLEMENTATION}

We have implemented the proposed solution on real sensor platform SensLAB. The SensLAB testbed is composed of 1024 nodes over 4 sites. In this paper we present only results on the 3 -dimensional grid composed of sensor node with wsn430 (cc1101) (Texas, 2011), (Chipcon, 2007). The electrical energy consumption is calculated through the linear energy model defined by the following equation:

$E=P_{1} t_{1}+P_{2} t_{2}+P_{3} t_{3}+\ldots \ldots .+P_{n-l} t_{n-1}+P_{n} t_{n}$; With $\mathrm{P}_{\mathrm{i}}$ : the average power consumption of node $i$ at the time $\mathrm{t}_{\mathrm{i}}$. For a five-hop communication model, Figure 2 shows the average energy evolution of 6 sensor nodes involved in the process. We found out that our proposed scheme has better energy consumption and does bring significant improvements.

\section{VERIFICATION AND VALIDATION}

We use the standard AVISPA (Armando, 2005) to validate our solution. Its ability to process protocols has been demonstrated through IETF (internet engineering task force) library protocol specified in High Level Protocol Specification (HLPSL) (Tseng, 2007). We checked secret property of the stored password, and strong authentication of LNs by the GW.

\subsection{Validation of Solution}

We check now the secrecy property of stored password and the strong authentication of the LNs by the GW.

- Secrecy property of the stored password: we must ensure that the stored password is kept confidential between the user, the LNs and the GW. This part involves in two different sessions the user, the GW, the LN and the intruder. The first session called here normal session consists of the legitimate user, the LN and the 
GW. The second session or intruder session involves the intruder playing the role of a user in order to attack the protocol.

$$
\begin{aligned}
& \text { - Normal session: User ( } u \text { - LN (l) - GW (g) } \\
& \text { - Intruder session: Intruder (i) - LN (l) - GW (g) }
\end{aligned}
$$

Figure 3 presents results achieved by AVISPA. We can see in the results that the protocol is declared SAFE, i.e no attacks returned.

- Secrecy and strong authentication: this must guarantee that the stored password is confidential and at the same time a strong authentication between the LNs and the GW. There are three different sessions. A normal session, a second session in which the intruder claims to be the user, and in the third session in which the intruder plays the role of the LN.

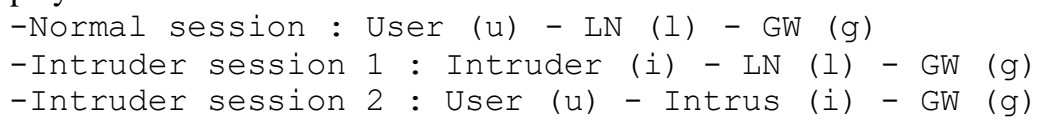

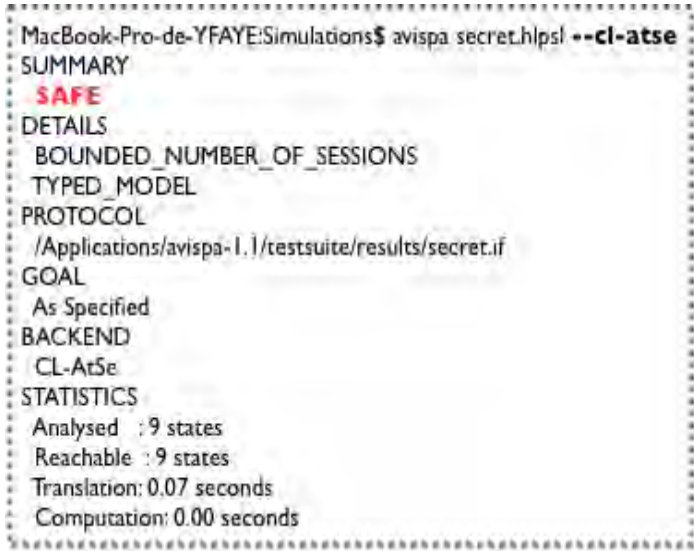

Figure 3. Verification of security properties of secret

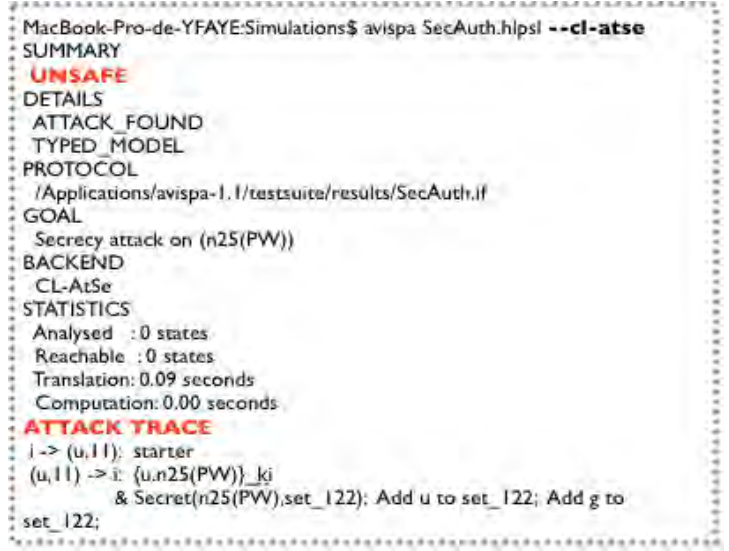

Figure 4. Verification of security properties of secret and authentication

\subsection{Discussion and Analysis of Results}

On Figure 4, the protocol is declared UNSAFE, i.e. not secure, and an attack is returned. An attack can take place without compromising security. After analyzing attack's traces, we see that the intruder claims to be a LN to the user according to the following events:

$$
i \rightarrow(u, 11) \text { : start. }
$$

The intruder $i$ initializes a session with the identifier (11) by sending a signal "start" composed of the identity of the user $u$, and the session (11) to the user so that the last one sends back to him an encrypted message with ki (key made by i and sent to u).

$(u, 11) \rightarrow\{u . n 25(P W)\} \_k i \&$ Secret $(u . n 25(P W)$, set_122), Add u to set_122, Add $g$ to set_122;

In the same session (11), the event $(\mathrm{u}, 11) \rightarrow\{\mathrm{u} . \mathrm{n} 25(\mathrm{PW})\}$ ki indicates that the user $\mathrm{u}$ sends back to the intruder his identity concatenated with the password n25 encrypted with the key ki. The fragment \& Secret(u.n25(PW), set 122) indicates that the PW identified by $\mathrm{n} 25$ is secret and is stored in variable set 122 which is generated automatically. Finally, the two events Add u to set 127 and Add g to set 122 indicate that only u (user) and g (gateway) are allowed to know the variable. In conclusion, an attack is conducted without success, the intruder $\mathrm{i}$, does not have the secret after this attempt.

\section{CONCLUSION}

In this paper, we have proposed a scheme to improve energy consumption by reducing the number of bits transmitted in Vaidya and al. (Vaidya, 2009), and in (Faye, 2012). The proposed technique is applicable to all solutions using the same approach with a very low probability of compromising security for a significant reduction of transmitted bits. Also, this technique can be easily applied to almost many existing user 
authentication schemes. The implementation on the SensLab plate shows that our scheme has better energy consumption. A verification and validation of the security properties with AVISPA justifies its resistance to the attack.

\section{REFERENCES}

Armando A. et al, 2005. The AVISPA tool for the automated validation of internet security protocols and applications", Computer Aided Verification, Springer, Berlin, pp 281-285.

Awasthi A. K. et al, 2003. A remote user authentication scheme using smart cards with Forward Secrecy. IEEE Transactions on Consumer Electronics, vol. 49, 4 pp.1246-1248.

Chevalier Y. et al, 2004. A high level protocol specification language for industrial security-sensitive protocols, Proc. SAPS, vol. 4, p. 193-205.

Chipcon , 2007, CC2420 2.4GHz IEEE 802.15.4 RF transceiver. http://www.ti.com/lit/gpn/ cc2420.

Das M. et al, 2004, A Dynamic ID-based Remote User Authentication Scheme, IEEE Transactions on Consumer Electronics, vol.50, 2. pp 629-631

Das K. et al, 2016, A secure and robust temporal credential-based three-factor user authentication scheme for wireless sensor networks. Peer-to-peer Networking and Applications, vol. 9, p. 223-244.

Faye Y. et al, 2011. A Survey of Access Control Schemes in Wireless Sensor Networks, World. Academy of Science, Engineering and Technology. Issue 59: 2011, Paris, France, pp 814-823,

Faye Y. et al, 2012. A user authentication-based prob-abilistic risk approach for Wireless Sensor Networks. In: Mobile and Wireless Networking (iCOST), International Conference on Selected Topics in. IEEE, 2012. p. 124-129.

Hwang M. et al, 2002. An E1Gamal-like cryptosystem for enciphering large messages, IEEE Trans .on Knowledge and Data Engineering, vol. 14, 2, pp.445-446.

Jiang Q. et al, 2015. An efficient two-factor user authentication scheme with unlinkability for wireless sensor networks. Peer-to-peer Networking and Applications, 6, vol. 8, p. 1070-1081.

Karp M. 1991. An introduction to randomized algorithms. DiscreteApplied Mathematics, 34(1-3), 165-201.

Kumari S. et al, 2015. User authentication schemes for wireless sensor networks: A review. Ad Hoc Networks, vol. 27, p. 159-194.

Krovetz T. et al, 2001. Fast Universal Hashing with Small Keys and No Preprocessing: The PolyR Construction. D. Won (Ed.): ICISC 2000, LNCS, Springer-Verlag Berlin Heidelberg, pp. 73-89

Lamport L., 1981. Password authentication with insecure communication, Communications of the ACM, 1981.Commun ACM, vol.24, no.11, pp.770-772

Lee C. et al, 2002. A remote user authentication scheme using hash functions, ACM Operating Systems Review, vol. 36, n4, pp.23-29.

Lee C. et al, 2005. An Improved Low Communication Cost User Authentication Scheme for Mobile Communication, Proceedings of the IEEE 19th International Conference on Advanced Information Networking and Applications, Taiwan.

Li X. et al, 2018. A three-factor anonymous authentication scheme for wireless sensor networks in internet of things environments. Journal of Network and Computer Applications, pp 194-204.

Ling C. et al, 2017. A Secure and Efficient One-time Password Authentication Scheme for WSN. IJ Network Security, 19(2), pp 177-181

Shen J. J. et al, 2003. A modified remote user authentication scheme using smart cards, IEEE Trans. on Consumer Electron. vol. 49, 2, pp.414-416.

Sun H., 2000. An Efficient remote user authentication scheme using smart cards, IEEE Trans. on Consumer Electron. vol. 46, 4, pp. 958-961.

TexasInstruments, 2011, CC1101 Low-Power Sub-1GHz RF Transceiver, .http://www.ti.com/lit/gpn/cc1101.

Tseng H. et al, 2007. An improved dynamic user authentication scheme for wireless sensor networks. In Proceedings of the IEEE Global Communications Conference (GLOBECOM07), pp 986-990.

Vaidya B. et al, 2009. Improved Robust User Authenti- cation Scheme for Wireless Sensor Networks. Wireless Communication and Sensor Networks (WCSN), Fifth IEEE Conference

Wong K. et al, 2006, A dynamic user authentication scheme for wireless sensor networks, In Proceedings of the IEEE International Conference on Sensor Networks, Ubiquitous, and Trustworthy Computing, vol. 1, pp. 244-251

Xue X. et al, 2013, A temporal-credential-basedmutual authentication and key agreement scheme for wireless sensor networks, Journal of Network and Computer Applications, 1, vol. 36, p. 316-323.

Zhang X. et al, 2018. , Xin, An novel anonymous user WSN authentication for Internet of Things. 'Soft Computing : 1-9. 